Pacific Journal of Mathematics

MINIMAL FIRST COUNTABLE HAUSDORFF SPACES 


\title{
MINIMAL FIRST COUNTABLE HAUSDORFF SPACES
}

\author{
R. M. Stephenson, JR.
}

If $\mathscr{P}$ is a property of topologies, a $\mathscr{P}$-space $(X, \mathscr{T})$ is called a $\mathscr{P}$-minimal space if there exists no $\mathscr{P}$-topology on $X$ properly contained in $\mathscr{T}$. Throughout the following, $\mathscr{H}=$ first countable and Hausdorff and $\mathscr{C}=$ first countable and completely Hausdorff (a space $X$ is called completely Hausdorff if the continuous real valued functions defined on $X$ separate the points of $X$ ).

In this paper we give examples of $\mathscr{H}$-minimal $\mathscr{C}$-spaces that are (i) not regular and (ii) regular but neither completely regular nor countably compact.

Two other results obtained are the following. (a) Every locally pseudocompact zero-dimensional $\mathscr{H}$-space can be embedded densely in a pseudocompact zero-dimensional $\mathscr{H}$ space. (b) Let $\mathscr{P}=\mathscr{C}$, completely regular $\mathscr{H}$, or zerodimensional $\mathscr{H}$, and suppose that $X$ is a $\mathscr{P}$-space such that for every $\mathscr{P}$-space $Y$ and continuous mapping $f: X \rightarrow Y, f$ is closed. Then $X$ is countably compact.

$N$ will denote the set of natural numbers, and $C(X, Y)$ will denote the family of continuous mappings of $X$ into $Y$. For definitions, see [4].

1. An embedding theorem and some examples. Recall that a space $(X, \mathscr{T})$ is said to be semiregular if $\{\stackrel{\circ}{T} \mid T \in \mathscr{T}\}$ is a base for $\mathscr{T}$. If $(X, \mathscr{T})$ has a property $\mathscr{P}$, then $(X, \mathscr{T})$ is said to be $\mathscr{P}$ closed provided that it is a closed subset of every $\mathscr{P}$-space in which it can be embedded.

For many properties $\mathscr{P}$, it is known that $\mathscr{P}$-minimal and $\mathscr{P}$ closed spaces are closely connected. For the case $\mathscr{P}=\mathscr{H}$, the following two results, established in [11], will be used below. An $\mathscr{H}$-space $X$ is $\mathscr{H}$-closed if and only if every countable open filter base on $X$ has nonempty adherence. An $\mathscr{C}$-space is $\mathscr{H}$-minimal if and only if it is semiregular and $\mathscr{H}$-closed.

We shall now describe constructions which can be used to densely embed certain $\mathscr{C}$-spaces in $\mathscr{H}$-minimal ( $\mathscr{C}$-closed) $\mathscr{C}$-spaces. As special cases, we shall obtain examples with the properties mentioned in the introduction. First some terminology is needed.

A space $X$ is said to be locally pseudocompact (W. W. Comfort) if every point of $X$ has a pseudocompact neighborhood. 
A filter base $\mathscr{F}$ is said to be pseudocompact if for every $F \in \mathscr{F}$ and $G \in \mathscr{F}, F-G$ is pseudocompact. $\mathscr{F}$ is called zero-dimensional if the sets belonging to it are open- and-closed.

Notation. (B. Banaschewski). Let $\mathscr{C l}$ be a family of open filter bases on a space $X$. Let $\{p(\mathscr{F}) \mid \mathscr{F} \in \mathscr{C}\}$ be a new set of distinct points, and let $X(\mathscr{C})$ be the space whose points are the elements of $X \cup\{p(\mathscr{F}) \mid \mathscr{F} \in \mathscr{C l}\}$ and whose topology has as a base sets of the form $V^{*}=V \cup\{p(\mathscr{F}) \mid V$ contains some member of $\mathscr{F}\}$, where $V$ is any open subset of $X$.

THEOREM 1.1. Let $X$ be an $\mathscr{H}$-space containing a point a such that $X-\{a\}$ is a zero-dimensional locally pseudocompact space. Let $\mathscr{N}=\{\mathscr{F} \mid \mathscr{F}$ is a free, countable, pseudocompact, zero-dimensional filter base on $X$, and denote by $\mathscr{L l}$ a maximal subset of $\mathscr{N}$ such that whenever $\mathscr{F}, \mathscr{G} \in \mathscr{M l}$ with $\mathscr{F} \neq \mathscr{G}$, then there exist disjoint sets $F \in \mathscr{F}$ and $G \in \mathscr{G}$.

Then the space $X(\mathscr{C l})$ is an $\mathscr{H}$-closed $\mathscr{C}$-space in which $X$ is embedded as a dense subset, and $X(\mathscr{L l})$ is $\mathscr{H}$-minimal if and only if $X$ is semiregular.

Proof. $X(\mathscr{C l})$ is clearly an $\mathscr{H}$-space. Furthermore, it follows from the hypothesis that each point of $X(\mathscr{C})-\{a\}$ has a fundamental system of feebly compact open neighborhoods. Thus the characteristic functions of open-and-closed subsets of $X(\mathscr{C})$ separate the points of $X(\mathscr{C l})$ and $X(\mathscr{C l})$ is a $\mathscr{C}$-space.

Suppose that $\mathscr{F}$ is a countable open filter base on $X(\mathscr{C})$ and no point of $X$ is an adherent point of $\mathscr{F}$. A slight modification of the proof of Lemma 2.17 in [11] shows that there exists a free, countable, pseudocompact, zero-dimensional filter base $\mathscr{G}$ on $X$ which is stronger than the filter base $\mathscr{F} \mid X$. By the maximality of $\mathscr{M}$, there exists $\mathscr{K} \in \mathscr{M}$ with $G \cap H$ nonempty for all $G \in \mathscr{G}$ and $H \in \mathscr{K}$. Thus $p(\mathscr{K})$ is an adherent point of $\mathscr{F}$.

To check semiregularity, it suffices to observe that if

$$
a \in V=\operatorname{Int}_{X} C l_{X} V \text {, then } V^{*}=\operatorname{Int}_{X(\mathcal{A})} C l_{X(\mathcal{N})} V^{*} .
$$

Theorem 1.2. Let $X$ and $a$ be as in Theorem 1.1, and suppose that $\left\{V_{n} \mid n \in N\right\}$ is a fundamental system of open neighborhoods for a such that $V_{1}=X$ and each $V_{n} \supset C l_{X} V_{n+1}$. Let $\mathscr{C l}$ be a maximal family of free, countable, pseudocompact, zero-dimensional filter bases on $X$ such that (a) whenever $\mathscr{F}, \mathscr{G} \in \mathscr{C}$ with $\mathscr{F} \neq \mathscr{G}$, then there 
exist disjoint sets $F \in \mathscr{F}$ and $G \in \mathscr{G}$, and (b) for every $\mathscr{F} \in \mathscr{C l}$ there exists $n \in N$ such that $\cup \mathscr{F}^{-} \subset V_{n}-V_{n+1}$.

Then $X(\mathscr{C l})$ is a regular $\mathscr{C}$-space that is $\mathscr{H}$-minimal and contains $X$ as a dense subspace. If each $V_{n}$ is closed in $X$, then $X(\mathscr{C l})$ is zero-dimensional.

Proof. Since $\{p(\mathscr{F}) \mid \mathscr{F} \in \mathscr{C l}\}-\{$ a $\}$ is a closed discrete subset of $X(\mathscr{C l})-\{a\}$, it follows from (b) that $C l_{X(\mathscr{M})} V_{n+1}^{*}=V_{n+1}^{*} \cup C l_{X} V_{n+1}$. Thus $X(\mathscr{C})$ is regular, and if each $V_{n}$ is closed in $X$, then $X(\mathscr{C l})$ is zero-dimensional.

The proof that $X(\mathscr{C l})$ is feebly compact is similar to the corresponding proof given for Theorem 1.1-one just notes that for some $n$, $\mathscr{F} \mid\left(C l_{X} V_{n}-C l_{X} V_{n+1}\right)$ is a filter base, and so $\mathscr{G}$ can be chosen with the property that $\cup \mathscr{G} \subset V_{n}-V_{n+1}$.

REMARK 1.3. In case the set $I$ of isolated points of $X$ is a dense subset of $X, \mathscr{C}$ can be defined as follows. Let $\mathscr{E}$ be a maximal family of countably infinite subsets of $I$ such that (a) the intersection of any two members of $\mathscr{E}$ is finite, and (b) each member of $\mathscr{E}$ is a closed subset of $X$ (for Theorem 1.2, a closed subset of some $\left.C l_{X}\left(V_{n}-V_{n+1}\right)\right)$. For each $E \in \mathscr{E}$ let $\mathscr{F}(E)$ be the complements in $E$ of finite subsets of $E$. Take $\mathscr{C}=\{\mathscr{F}(E) \mid E \in \mathscr{E}\}$.

REMark 1.4. For the case $X=N$ and $\mathscr{C l}$ infinite, the space $X(\mathscr{C})$ is due to J. Isbell (see $[5,5 \mathrm{I}]$ ).

REMARK 1.5. In general, the space $X(\mathscr{C l})$ is not countably compact and hence not weakly normal, for each $\{p(\mathscr{F}) \mid \mathscr{F} \in \mathscr{C}\}-V^{*}$ is a closed discrete subset of $X(\mathscr{M})$.

COROLLARY 1.6. Every locally pseudocompact zero-dimensional $\mathscr{H}$-space can be embedded densely in a pseudocompaet zero-dimensional $\mathscr{H}$-space.

ExAMPle 1.7. For the following $X$, the space $X(\mathscr{C l})$ is an $\mathscr{H}$-minimal $\mathscr{C}$-space that is not regular.

Let $T=\{0\} \cup\{1 / n \in N\}$, with the usual topology, choose a point a not in the product space $N \times T$, and let $X=\{a\} \cup(N \times T)$, topologized as follows: every open subset of $N \times T$ is open in $X$; a neighborhood of $\mathrm{a}$ is any set of the form $V_{n}=\{a\} \cup\{(x, y) \in X \mid x \geqq n$ and $1 / y$ is an 
even integer $\}, n \in N . \quad$ ( $X$ is homeomorphic to $E-\{b\}$, where $E$ is as in $[13$, p. 268].)

One can take $\mathscr{l}$ to be a maximal family of infinite subsets of $X-C l V_{1}$ such that the following hold:

(i) For all $M, M^{\prime} \in \mathscr{A}, M \neq M^{\prime}$ implies $M \cap M^{\prime}$ is finite;

(ii) For all $M \in \mathscr{C}$ and $n \in N, M \cap(\{n\} \times T)$ is finite.

ExAmple 1.8. For the following $X$, the space $X(\mathscr{C})$ (of Theorem $1.2)$ is an $\mathscr{H}$-minimal $\mathscr{C}$-space that is regular but not completely regular.

Let $Y$ be the set of ordinal numbers less than the first uncountable ordinal, with the order topology, let $M$ be the set of limit ordinals in $Y$, and denote $Y-M$ by $I$. Let $Z=I \times\{0\} \cup Y \times N$, topologized as follows: $Y \times N$ has the product topology, and $Y \times N$ is open in $Z$; a neighborhood of a point $(i, 0) \in Z$ is any subset of $Z$ that contains $(i, 0)$ and all but finitely many elements of $\{i\} \times N$. Let $L$ and $R$ denote the product spaces $Z \times\{1\}$ and $Z \times\{2\}$, and set $U=L \cup R$, with the weak topology generated by $\{L, R\}$. Let $S$ be the relation on $U$ defined by the rule: $(x, i, j) S(y, k, n)$ if (a) $x=y, i=k$, and $j=n$, or (b) $x=y \in M$ and $i=k$. Denote the quotient space $U / S$ by $T$. We shall continue to use the symbols $(x, i, j)$ for the points of $T$.

On the product space $T \times N$ define $(t, n) W\left(t^{\prime}, n^{\prime}\right)$ if (a) $t=t^{\prime}$ and $n=n^{\prime}$, or (b) $t=(x, 0, j), t^{\prime}=\left(x, 0, j^{\prime}\right)$, and $n^{\prime}-n=j-j^{\prime}=1$ or $n-n^{\prime}=j^{\prime}-j=1$. Let $V$ be the quotient space $(T \times N) / W$. Choose a new point $a$ and let $X=V \cup\{a\}$, topologized as follows: every open subset of $V$ is open in $X$; a neighborhood of $a$ is any set of the form $V_{n}=\{a\} \cup\{(t, m) \in V \mid m \geqq n\}, n \in N$.

It is not difficult to see that $X$ is a first countable regular space whose isolated points are dense, and $X-\{\alpha\}$ is zero-dimensional and locally compact. $X$ is not completely regular, because for every $\mathrm{f} \in C(X)$ there exists $m \in Y$ such that $f$ is constant on

$$
\{(x, 0, j, n) \mid x \geqq m, j=1 \text { or } j=2 \text {, and } n \in N\} \text {. }
$$

Thus $V_{2}$, for example, contains no zero set neighborhood of $a$.

REMARK 1.9. The construction above is a modification of Tychonoff's regular but not completely regular space [12].

In [7] F. B. Jones has constructed a $\mathscr{C}$-space that is not com- 
pletely regular but that is a Moore space. His space cannot be used here, however, because it is neither locally pseudocompact nor zerodimensional.

In the literature there are many less messy examples of $\mathscr{C}$-closed or $\mathscr{H}$-minimal spaces that are not regular; however, the author does not know of any $\mathscr{C}$-minimal space appearing elsewhere that is not regular (or completely regular).

REMARK 1.10. If one glues together (as in [2]) two copies of the space in Example 1.8, then one gets an example of a regular $\mathscr{H}$ minimal space that is not completely Hausdorff.

2. $\mathscr{C}$-minimal spaces and closed mappings. If $\mathscr{P}$ denotes any one of the usual separation properties, it is known that every $\mathscr{P}$-minimal completely Hausdorff space is compact (e.g., see [6]). Moreover C. T. Scarborough [9] has observed that a completely Hausdorff-minimal space is compact.

One might then expect $\mathscr{C}$-minimal spaces to be well behaved, to be, say, at least countably compact. Of course, Isbell's example or Mrówka's [8] (or ours) shows that this is not the case. The following characterization theorems may, therefore, be of interest.

Definition. (H. E. Hayes) An open filter base $\mathscr{F}$ on a space $X$ is said to be completely Hausdorff provided that for every $x \in X$, if $x$ is not an adherent point of $\mathscr{F}$, then there exist $f \in C(X)$ and $F \in \mathscr{F}$ such that $f(F)=0$ and $f(x)=1$.

Using usual techniques, one can prove the following.

THEOREM 2.1. Let $X$ be a $\mathscr{C}$-space. The following are equivalent.

(i) $X$ is $\mathscr{C}$-closed.

(ii) Every countable completely Hausdorff filter base on $X$ has an adherent potnt.

(iii) For every $\mathscr{C}$-space $Y$ and $f \in C(X, Y), f(X)$ is $\mathscr{C}$-closed.

In order to obtain a $\mathscr{C}$-analogue of Theorem 2.4 of [11], we need a second definition.

Definition. An open filter base $\mathscr{F}$ on a space $X$ is said to be almost completely Hausdorff if there exists $p \in X$ so that for every $x \in X-\{p\}$, if $x$ is not an adherent point of $\mathscr{F}$, then there exist $f \in C(X)$ and $F \in \mathscr{F}$ such that $f(F)=0$ and $f(x)=1$. 
TheOREM 2.2. Let $X$ be a $\mathscr{C}$-space. The following are equivalent.

(i) $X$ is $\mathscr{C}$-minimal.

(ii) Every countable completely Hausdorff filter base on $X$ that has a unique adherent point is convergent.

(iii) $X$ is semiregular, and every countable almost completely Hausdorff filter base on $X$ has an adherent point.

The proof is somewhat similar to the proofs needed for Theorems 2.4 and 2.9 in [11].

The next result, to be contrasted with (iii) of Theorem 2.1, is a partial converse to the following well-known theorem: If $X$ is a countably compact space, $Y$ is an $\mathscr{H}$-space (or a space of the type $E_{1}$ studied in [1]), and $f \in C(X, Y)$, then $f$ is closed.

We shall call an open filter base $\mathscr{F}$ on $X$ completely regular if for each $F \in \mathscr{F}$ there exist $G \in \mathscr{F}$ and $f \in C(X,[0,1])$ such that $f$ vanishes on $G$ and equals 1 on $X-F$.

Theorem 2.3. Let $\mathscr{P}$ denote either completely Hausdorff, completely regular, or zero-dimensional, and suppose that $X$ is a $\mathscr{P}$-space which is also an $\mathscr{H}$-space. The following are equivalent.

(i) $X$ is countably compact.

(ii) For every $\mathscr{H}$-space $Y$ and $f \in C(X, Y), f$ is closed.

(iii) For every $\mathscr{P}$-space $Y$ that is an $\mathscr{H}$-space and $f \in C(X, Y)$, $f$ is closed.

(iv) For every closed subset $C$ of $X$ and every countable $\mathscr{P}$-filter base $\mathscr{F}$ on $X$, if $\mathscr{F} \mid C$ is a filter base and if $\cap \mathscr{F}=\cap\{\bar{F} \mid F \in \mathscr{F}\}$, then there is a point $c \in C$ which is in $\cap \mathscr{F}$.

Proof. (i) $\Rightarrow$ (ii) is known. (ii) $\Rightarrow$ (iii) is obvious. A proof not too different from one in [3] shows that (iii) $\Leftrightarrow$ (iv). We shall prove that (iv) $\Rightarrow$ (i) for the case $\mathscr{P}=$ completely Hausdorff.

Let us suppose then that $X$ is a $\mathscr{C}$-space which contains a countably infinite closed discrete subset $C$.

Consider a point $c \in C$. Since $X$ is completely Hausdorff and $C-\{c\}$ is countable, there exists $f \in C(X)$ for which $f(c) \notin f(C-\{c\})$. Since $C-\{c\}$ is a closed subset of $X$ and $f$ is closed, we can choose $g \in C((-\infty, \infty))$ with $g(f(c))=1$ and $g(f(C-\{c\}))=0$. Set $h_{c}=g \circ f$.

Let $\mathscr{F}$ be the family of all finite intersections of

$$
\left\{h_{c}^{-1}(-1 / n, 1 / n) \mid n \in N \text { and } c \in C\right\} \text {. }
$$


Then it is easy to see that $\mathscr{F}$ is a countable completely regular (and hence completely Hausdorff) filter base on $X$, that $\cap \mathscr{F}=\cap\{\bar{F} \mid F \in \mathscr{F}\}$, and that $\mathscr{F} \mid C$ is a filter base. On the other hand, one also has $C \cap \cap \mathscr{F}=\dot{\phi}$. This contradicts (iv).

REMARK 2.4. There exists an $\mathscr{C}$-space $X$ that is not countably compact but which has the property: for every Hausdorff space $Y$ and $f \in C(X, Y), f$ is closed. See [3] and [14].

\section{REFERENCES}

1. C. E. Aull, A certain class of topological spaces, Prace Mat. 11 (1967), 49-53.

2. M. P. Berri and R. H. Sorgenfrey, Minimal regular spaces, Proc. Amer. Math. Soc., 14 (1963), 454-458.

3. R. F. Dickman, Jr. and Alan Zame, Functionally compact spaces, Pacific J. Math., 31 (1969), 303-311.

4. J. Dugundji, Topology, Allyn and Bacon, Boston, Mass., 1966.

5. L. Gillman and M. Jerison, Rings of continuous functions, Van Nostrand, New York, 1960.

6. H. Herrlich, Tע-Abgeschlossenheit und Tע-Minimalität, Math. Z., 88(1965), 285-294.

7. F. B. Jones, Moore spaces and uniform spaces, Proc. Amer. Math. Soc., 9 (1958), 483-486.

8. S. Mrówka, On completely regular spaces, Fund. Math., 41 (1954), 105-106.

9. C. T. Scarborough and R. M. Stephenson, Jr., Minimal topologies, Colloq. Math., 19 (1968), 215-219.

10. C. T. Scarborough and A. H. Stone, Products of nearly compact spaces, Trans. Amer. Math. Soc., 124 (1966), 131-147.

11. R. M. Stephenson, Jr., Minimal first countable topologies, Trans. Amer. Math. Soc., 138 (1969), 115-127.

12. A. Tychonoff, Über die topologische Erweiterung von Rüumen, Math. Ann., 102 (1930), 544-561.

13. P. Urysohn, Über die Machtigkeit der zusammenhängenden Mengen, Math. Ann., 94 (1925), 262-295.

14. Giovanni A. Viglino, C-Compact spaces, Duke Math. J., 36 (1969), 761-764.

Received February 17, 1970. This research was partially supported by a grant from the University of North Carolina.

University of North Carolina at Chapel Hill 



\title{
PACIFIC JOURNAL OF MATHEMATICS
}

\section{EDITORS}

H. SAMELSON

Stanford University

Stanford, California 94305

C. R. HobвY

University of Washington

Seattle, Washington 98105

\section{J. DugunduI}

Department of Mathematics

University of Southern California

Los Angeles, California 90007

RICHARD ARENS

University of California

Los Angeles, California 90024

\section{ASSOCIATE EDITORS}
E. F. BECKENBACH
B. H. Neumann
F. WOLE
K. Yoshida

\section{SUPPORTING INSTITUTIONS}

\author{
UNIVERSITY OF BRITISH COLUMBIA \\ CALIFORNIA INSTITUTE OF TECHNOLOGY \\ UNIVERSITY OF CALIFORNIA \\ MONTANA STATE UNIVERSITY \\ UNIVERSITY OF NEVADA \\ NEW MEXICO STATE UNIVERSITY \\ OREGON STATE UNIVERSITY \\ UNIVERSITY OF OREGON \\ OSAKA UNIVERSITY \\ UNIVERSITY OF SOUTHERN CALIFORNIA
}

\author{
STANFORD UNIVERSITY \\ UNIVERSITY OF TOKYO \\ UNIVERSITY OF UTAH \\ WASHINGTON STATE UNIVERSITY \\ UNIVERSITY OF WASHINGTON

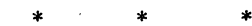 \\ AMERICAN MATHEMATICAL SOCIETY \\ CHEVRON RESEARCH CORPORATION \\ NAVAL WEAPONS CENTER
}

The Supporting Institutions listed above contribute to the cost of publication of this Journal, but they are not owners or publishers and have no responsibility for its content or policies.

Mathematical papers intended for publication in the Pacific Journal of Mathematics should be in typed form or offset-reproduced, (not dittoed), double spaced with large margins. Underline Greek letters in red, German in green, and script in blue. The first paragraph or two must be capable of being used separately as a synopsis of the entire paper. The editorial "we" must not be used in the synopsis, and items of the bibliography should not be cited there unless absolutely necessary, in which case they must be identified by author and Journal, rather than by item number. Manuscripts, in duplicate if possible, may be sent to any one of the four editors. Please classify according to the scheme of Math. Rev. Index to Vol. 39. All other communications to the editors should be addressed to the managing editor, Richard Arens, University of California, Los Angeles, California, 90024.

50 reprints are provided free for each article; additional copies may be obtained at cost in multiples of 50 .

The Pacific Journal of Mathematics is published monthly. Effective with Volume 16 the price per volume (3 numbers) is $\$ 8.00$; single issues, $\$ 3.00$. Special price for current issues to individual faculty members of supporting institutions and to individual members of the American Mathematical Society: $\$ 4.00$ per volume; single issues $\$ 1.50$. Back numbers are available.

Subscriptions, orders for back numbers, and changes of address should be sent to Pacific Journal of Mathematics, 103 Highland Boulevard, Berkeley, California, 94708.

PUBLISHED BY PACIFIC JOURNAL OF MATHEMATICS, A NON-PROFIT CORPORATION

Printed at Kokusai Bunken Insatsusha (International Academic Printing Co., Ltd.), 7-17, Fujimi 2-chome, Chiyoda-ku, Tokyo, Japan. 


\section{Pacific Journal of Mathematics}

\section{Vol. 36, No. $3 \quad$ BadMonth, 1971}

E. M. Alfsen and B. Hirsberg, On dominated extensions in linear subspaces of

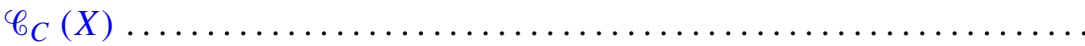

Joby Milo Anthony, Topologies for quotient fields of commutative integral

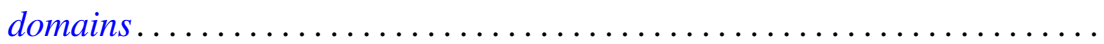

V. Balakrishnan, G. Sankaranarayanan and C. Suyambulingom, Ordered cycle lengths in a random permutation .......................... 603

Victor Allen Belfi, Nontangential homotopy equivalences............... 615

Jane Maxwell Day, Compact semigroups with square roots .............. 623

Norman Henry Eggert, Jr., Quasi regular groups of finite commutative nilpotent algebras .......................................... 631

Paul Erdős and Ernst Gabor Straus, Some number theoretic results ......... 635

George Rudolph Gordh, Jr., Monotone decompositions of irreducible Hausdorff continua .............................................

Darald Joe Hartfiel, The matrix equation $A X B=X \ldots \ldots \ldots \ldots \ldots \ldots . \ldots 69$

James Howard Hedlund, Expansive automorphisms of Banach spaces. II . . . . 671

I. Martin (Irving) Isaacs, The p-parts of character degrees in p-solvable

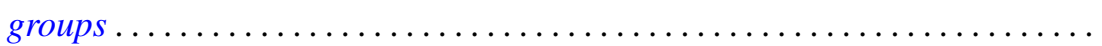

Donald Glen Johnson, Rings of quotients of $\Phi$-algebras ............... 693

Norman Lloyd Johnson, Transition planes constructed from semifield planes....

Anne Bramble Searle Koehler, Quasi-projective and quasi-injective modules.

James J. Kuzmanovich, Completions of Dedekind prime rings as second endomorphism rings...

B. T. Y. Kwee, On generalized translated quasi-Cesàro summability ...

Yves A. Lequain, Differential simplicity and complete integral closure

741

Mordechai Lewin, On nonnegative matrices.

753

Kevin Mor McCrimmon, Speciality of quadratic Jordan algebras ...

Hussain Sayid Nur, Singular perturbations of differential equations in abstract spaces .....................................

D. K. Oates, A non-compact Krein-Milman theorem .

Lavon Barry Page, Operators that commute with a unilateral shift on an invariant subspace...

Helga Schirmer, Properties of fixed point sets on dendrites.

Saharon Shelah, On the number of non-almost isomorphic models of $T$ in a

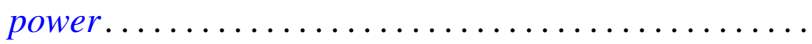

Robert Moffatt Stephenson Jr., Minimal first countable Hausdorff spaces....

Masamichi Takesaki, The quotient algebra of a finite von Neumann algebra 